\title{
Robust MPC Method for BMI Based Wheelchair
}

\author{
Tohru Kawabe \\ Department of Computer Science, University of Tsukuba, Tsukuba, Japan \\ E-mail:kawabe@cs.tsukuba.ac.jp \\ Received August 12, 2011; revised September 1, 2011; accepted September 28, 2011
}

\begin{abstract}
In this paper, robust MPC (Model Predictive Control) with adaptive DA converter method for the wheelchair using EEG (Electroencephalogram) based BMI (Brain Machine Interface) is discussed. The method is developed to apply to the obstacle avoidance system of wheelchair. This paper is the 1st stage for the development of the BMI based wheelchair in practical use. The robust MPC method is realized by using the minimax optimization with bounded constraint conditions. Some numerical examples are also included to demonstrate the effectiveness of the proposed methods the former stage of the real experiments.
\end{abstract}

Keywords: Wheelchair, Brain Machine Interface, Model Predictive Control, Adaptive DA Converter, Minimax Optimization

\section{Introduction}

In last few decades, MPC (Model Predictive Control) has been widely accepted in the industry. In the standard MPC formulation, the current control action is obtained by solving a finite or infinite horizon quadratic cost problem at every sample time using the current state of the plant as the initial state [1].

One of the significant merits of MPC is easy handling of constraints during the design and implementation of the controller. Conventionally, MPC has been used for systems with relatively slow-moving dynamics, for example, chemical or industrial processes and so on.

However, recent dramatic improvement of computer performance has made it possible to apply MPC to the continuous-time objects with fast-moving dynamics. The digital MPC method is now effective to control for various kinds of continuous-time objects. Such systems, the continuous-time objects controlled by discrete-time controller, are so-called the sampled-data control systems.

The analog-to-digital (AD) and the digital-to-analog (DA) conversions of signals are indispensable operations in the sampled-data control systems. The zero-order hold is usually used for the DA conversion on the assumption that the analog signals in each sampling interval are considered as constant values [2].

To improve the performance of sampled-data control systems, it's very important to take account of the behavior of systems in the sampling intervals. On this issue, some notable methods to design the discrete-time con- troller for continuous-time objects with $\mathrm{AD} / \mathrm{DA}$ conversion have been proposed [3-5]. Although the information about future sampling points is need for getting the current control input by the interpolating operations of the sampled-data control systems, it's impossible to obtain them. Therefore, we have been forced to tolerate the long time-delay during the DA conversion to wait for getting the indispensable information. In MPC algorithm, a prediction of the future system status is executed, and future control inputs based on the prediction are also calculated in each step. These future control inputs based on the prediction, therefore, are used for interpolation by sampling function. This idea is realized as an adaptive DA converter which can switch the sampling functions in each interval optimally according to the system status. It can realize the interpolation of samples in DA without long time-delay.

One of the drawback of MPC is explicitly lack of robust property with respect to model uncertainties or disturbances since the on-line minimized cost function is defined in terms of the nominal systems. A possible strategy for robust MPC is solving the so-called minimax problem [6,7], namely minimization problem over the control input of the robust performance measure maximized by plant uncertainties or disturbances. Some early works on robust MPC was proposed by Campo and Morari [8], and further developed by Zheng and Morari [9] for SISO FIR plants. Kothare solves minimax MPC problems with state-space uncertainties through LMIs [10]. Cuzzola improves the Kothare's method [10] to 
reduce conservativeness in [11]. Several methods of minimax MPC for systems with model uncertainties or disturbances can be found in $[12,13]$.

There has been some works of minimax MPC for systems with external disturbances in [14-16]. These methods are, however, based on infinite horizon quadratic cost functions, since it is rather hard to solve the minimax finite quadratic cost problems. The issue of minimax robust MPC therefore still deserves further attention $[14,17]$.

In this paper, minimax robust finite MPC method with the adaptive DA converter for an obstacle avoidance system of the robotic wheelchair using EEG (Electroencephalogram) based BMI (Brain Machine Interface) [18, 19] is discussed. This paper is the 1st stage for the development of the BMI based wheelchair in practical use.

The obstacle avoidance system of the wheelchair using EEG based BMI is one of the significant example of sampled-data controlled man-machine systems. One of the important points of the system is robustness property against the model uncertainties and disturbances. Since it is severely required that the system be always safe under whatever condition.

In the man-machine systems, it's key to unite man's judgment/recognition and the automatic control of the machine well. In this point, one of the key method is EEG based BMI. Since it can support to communicate for physically handicapped patients. The EEG based BMI is now in the process of reaching practical use for man-machine systems. The EEG signals of brain waves are considered to use as the urgent evasion signals for the obstacle avoidance system of wheelchair in this research. Some numerical examples are also included. The results give us the effectiveness of the system designed by the proposed method as the former stage of the real experiments.

This paper is organized as follows. In Section 2, minimax MPC problem is formulated. In Section 3, solving method of the problem is shown. In Section 4, MPC with adaptive DA converter is explained. In Section 5, experimental results of application of BMI based wheelchair are given. Finally, in Section 6, concluding remarks and future works are stated.

\section{BMI Based Wheelchair}

\subsection{BMI Using EEG}

The targeted system is as shown in Figure 1. It's one of the man-machine systems. In the man-machine systems, one of the most important point is to unite man's judgment, recognition, and the automatic control of the machine well. In this point, one of the key methods is BMI (Brain machine interface). Although the BMI has been used to support to communicate for physically handicapped patients, for example, ALS (Amyotrophic Lateral Sclerosis) or spinal cord injury, and so on.

EEG (Electroencephalogram) based BMI is now expect to be practical use for man-machine systems [18, 19]. One of the most significant man-machine systems

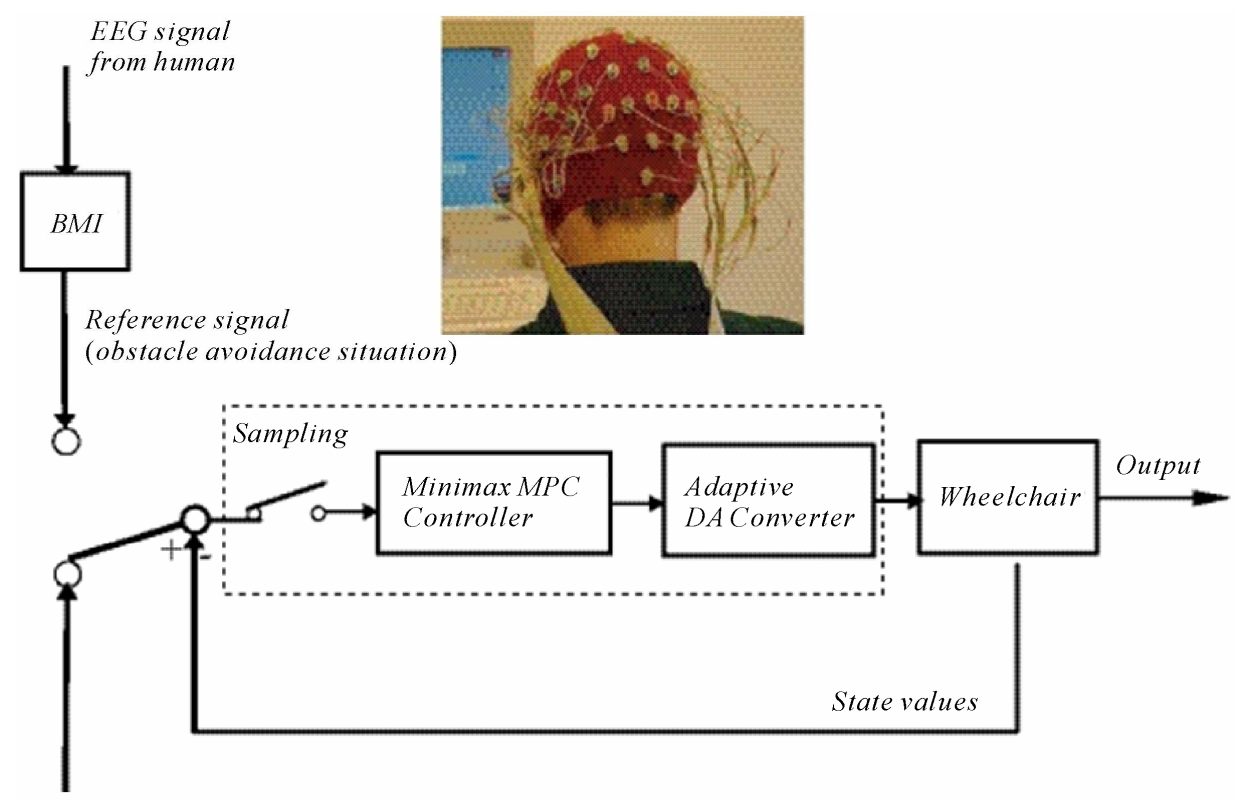

Reference signal (normal situation)

Figure 1. Targeted system. 
for handicapped persons is a wheelchair. Therefore, it's important to develop safely obstacle avoidance system of wheelchair.

In this research, the EEG signals of brain waves are considered to use as the urgent evasion signals for the obstacle avoidance system of robotic wheelchair. Generally, the EEG signals include redundant information that is unnecessary for decoding the commands and may also weaken the generalization performance of the classifier. To cope with this issue, Lal proposed a search method of better combinations of EEG channels by using a feature selection technique called RFE (Recursive Feature Elimination) [20]. Millan applied feature selection using decision trees to EEG data [21]. We have also developed the feature selection method based on the k-SVM (kernel Support Vector Machines) [22,23] with the backward stepwise selection for the BMI. This method can remove unnecessary or redundant features of EEG signals and keep only effective features for the classification task as a way of improving accuracy and quickness.

The combination of features that gives the largest evaluation value is considered the best (sub-optimal) combination of features. Since the urgent evasion signals are relevant to areas of the central part of the cerebrum cortex such as pre motor cortex, motor cortex and sensorimotor cortex, EEG signals were recorded from13 electrodes $(\mathrm{Fz}, \mathrm{FCz}, \mathrm{FC} 1, \mathrm{FC} 2, \mathrm{Cz}, \mathrm{C} 1, \mathrm{C} 2, \mathrm{C} 3, \mathrm{C} 4, \mathrm{CPz}$, $\mathrm{CP} 1, \mathrm{CP} 2, \mathrm{Pz})$ as shown in Figure $2(\mathrm{Fz}, \mathrm{FCz}, \mathrm{Cz}, \mathrm{CPz}$ and $\mathrm{Pz}$ are on the longitudinal fissure. $\mathrm{Cz}, \mathrm{C} 1, \mathrm{C} 2, \mathrm{C} 3$, $\mathrm{C} 4$ areon the central sulcus).

The power spectrum densities for eachelectrode was estimated using the Welch period gram and was divided into 12 components with a $2 \mathrm{~Hz}$ resolution. The resulting 156 features (13 channels times 12 components) were used as the initial set of features for the classifier.

\subsection{Wheelchair Model}

The wheel chair has two motors which rotate independ- ently. Although there are many control methods using velocities and angular velocities as manipulated variables [24], the dynamic model of the robot is used in this paper. Therefore, motor torques are set as manipulated variables [25], then the robot is torque-controlled and has two independent inputs.

We assume the center of gravity (C.G.) of the robot corresponds to center of the two wheels, and let the position of C.G. sets $(x, y)$, and $\theta$ denotes robot's direction (see Figure 3). The dynamic model of robot can be described following state space model [26] as follows.

$$
\left(\begin{array}{c}
\dot{v} \\
\dot{\omega}
\end{array}\right)=\left(\begin{array}{cc}
a_{1} & 0 \\
0 & a_{2}
\end{array}\right)\left(\begin{array}{l}
v \\
\omega
\end{array}\right)+\left(\begin{array}{cc}
b_{1} & b_{1} \\
b_{2} & -b_{2}
\end{array}\right)\left(\begin{array}{l}
u_{r} \\
u_{l}
\end{array}\right)
$$

where

$$
\begin{array}{ll}
a_{1}=\frac{-2 c}{M r^{2}+2 I_{\omega}}, & a_{2}=\frac{-2 c l^{2}}{I_{v} r^{2}+2 I_{\omega} l^{2}} \\
b_{1}=\frac{r}{M r^{2}+2 I_{\omega}}, & b_{2}=\frac{r l}{I_{v} r^{2}+2 I_{\omega} l^{2}}
\end{array}
$$

Controlled variable $v$ and $\omega$ are the velocity of C.G. and angular velocity respectively, $u_{r}$ and $u_{l}$ is right and left motors torques. The definition of parameters is shown in Table 1. The relation between $(v, \omega)$ and $(x, y, \theta)$ is described;

$$
\dot{x}=v \cos \theta, \dot{y}=v \sin \theta, \dot{\theta}=\omega
$$

Input torques $u_{r}$ and $u_{l}$ change $v$, and $\omega$ according to Equation (1), $v$ and $\omega$ change $x, y$ and $\theta$ according to Equation (2), too.

\section{Minimax Robust MPC Problem}

The target system in this paper is the sampled-data system. Hence, the control object is continuous-time system and the controller is designed in discrete-time. Then, let's consider the following general discrete-time model with uncertainties and disturbance.

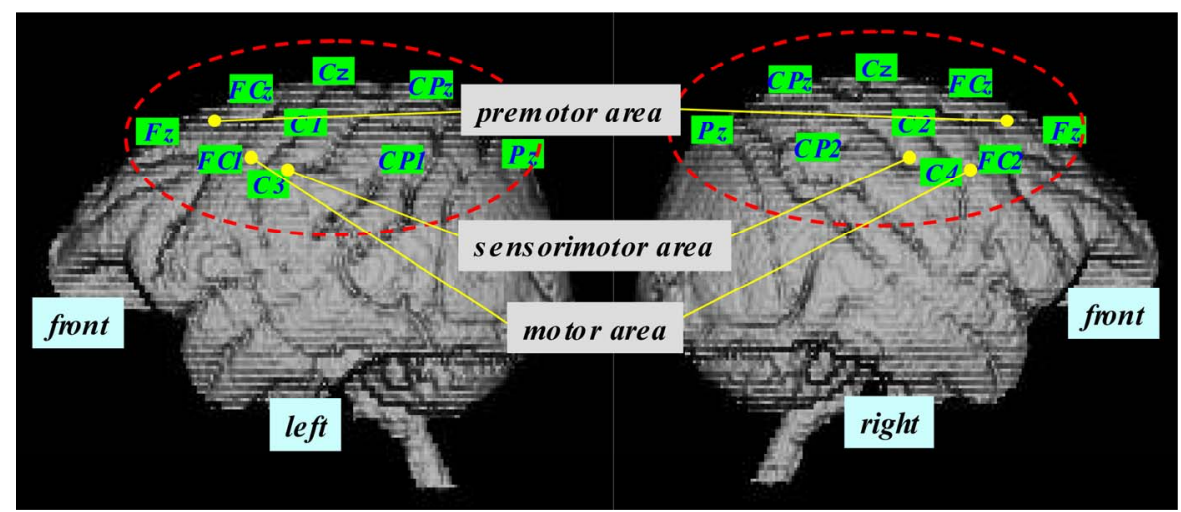

Figure 2. Location of the EEG electrodes in cerebrum. 


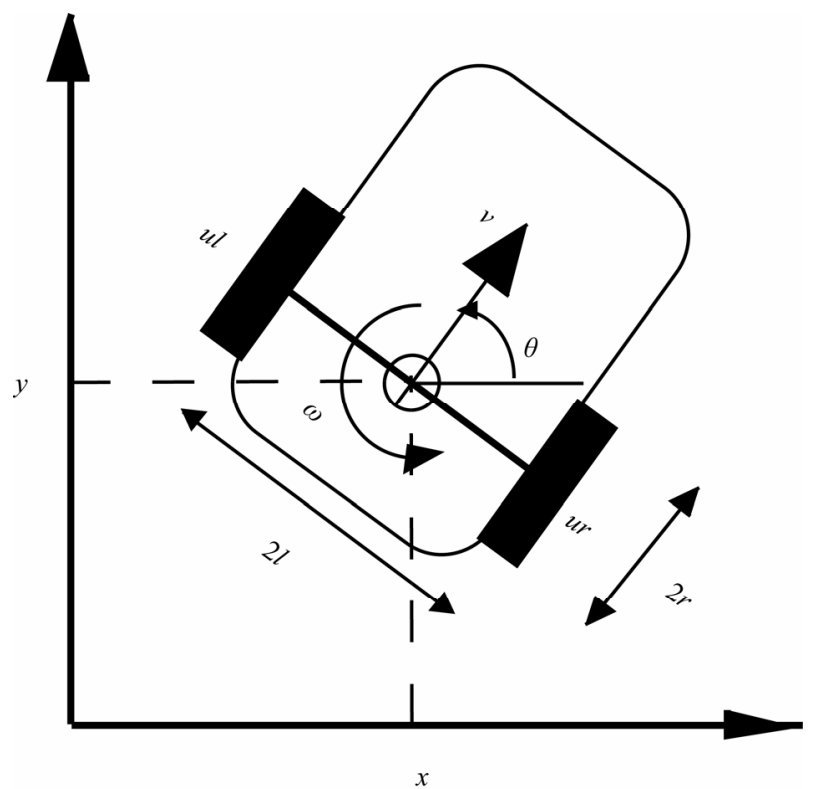

Figure 3. Model of robotic wheelchair.

Table 1. Definition of parameters.

\begin{tabular}{cc}
\hline$I_{\omega}$ & Inertia moment $\left[\mathrm{Nms}^{2} / \mathrm{rad}\right]$ \\
$M$ & Weight $[\mathrm{Kg}]$ \\
$I_{v}$ & Inertia moment of rotation center $\left[\mathrm{Nms}^{2} / \mathrm{rad}\right]$ \\
$l$ & Distance between wheel and rotation center $[\mathrm{m}]$ \\
$c$ & Viscosity coefficient of friction $\left[\mathrm{Nms}^{2} / \mathrm{rad}\right]$ \\
$r$ & Wheel radius $[\mathrm{m}]$ \\
\hline
\end{tabular}

$$
\begin{gathered}
x(k+1)=\left(A+L \Delta R_{A}\right) x(k)+\left(B+L \Delta R_{B}\right) u(k) \\
y(k)=C x(k)+\eta(k)
\end{gathered}
$$

where $x(k), u(k), y(k)$ and $\eta(k)$ denote the state, input, measure doutput and disturbance vector respectively, and where $\Delta$ is a diagonal structured uncertain parameters matrix satisfied $\Delta^{T} \Delta \leq I . L, R_{A}$ and $R_{B}$ are constant matrices. All these vectors and matrices have appropriate dimensions. Then, we can transform this system as

$$
\begin{gathered}
x(k+1)=A x(k)+B u(k) \\
z(k)=R_{A} x(k)+R_{B} u(k) \\
y(k)=C x(k)+\eta(k)
\end{gathered}
$$

where $w(k)(:=\Delta z(k))$. We assumed that the system is constrained with following conditions;

$$
\begin{gathered}
w^{T}(k+j) P_{w} w(k+j) \leq 1 \\
\eta^{T}(k+j) P_{\eta} \eta(k+j) \leq 1
\end{gathered}
$$

$$
\begin{gathered}
u^{T}(k+j) P_{u} u(k+j) \leq 1 \\
(j=0, \cdots, N-1)
\end{gathered}
$$

where $P_{w}, P_{\eta}, P_{u}$ are positive symmetric matrices for weights of constraints. For this system, the quadratic performance measure with finite horizon with positive weighting constant matrices $Q$ and $R$ as :

$$
J(k)=\sum_{j=0}^{N-1}\left\{\|y(k+j+1 \mid k)\|_{Q}^{2}+\|u(k+j \mid k)\|_{R}^{2}\right\}
$$

is used. $x(k+j \mid k), y(k+j \mid k)$ and $u(k+j \mid k)$ are the predicted state of the plant, the predicted output of the plant and the future control input at time $k+j$ respectively. Then, the design problem is formulated as the following minimax optimization problem.

$$
\min _{u(k+j \mid k)} \max _{w(k+j \mid k), \eta(k+j k)} J(k)
$$

subject to

$$
\begin{gathered}
w^{T}(k+j) P_{w} w(k+j) \leq 1 \\
\eta^{T}(k+j) P_{\eta} \eta(k+j) \leq 1 \\
u^{T}(k+j) P_{u} u(k+j) \leq 1 \\
(j=0, \cdots, N-1)
\end{gathered}
$$

Since the saddle point may not exist in general, it is difficult to solve this problem. Hence, the objective is to eliminate the maximization procedure and transform this problem to simple minimization problem which can be solved easily.

\section{Transformation of the Problem}

At each step $k$ the following state feedback is employed;

$$
u(k+j \mid k)=-F_{k+j} x(k+j \mid k)
$$

where $F_{k+j}$ is a feedback gain matrix. Then, introducing the following vectors

$$
\begin{aligned}
X & :=[x(k+1 \mid k) \cdots x(k+N \mid k)]^{T} \\
Y & :=[y(k+1 \mid k) \cdots y(k+N \mid k)]^{T} \\
U & :=[u(k \mid k) \cdots u(k+N-1 \mid k)]^{T} \\
W & :=[w(k \mid k) \cdots w(k+N-1 \mid k)]^{T} \\
\Lambda & :=[\eta(k \mid k) \cdots \eta(k+N-1 \mid k)]^{T}
\end{aligned}
$$

and using state space equation, Equations (5)-(7), recursively, we can derive 


$$
\begin{array}{r}
X=\tilde{A} x(k)+\tilde{B} U+\tilde{L} W \\
Y=C \tilde{A} x(k)+C \tilde{L} W+\Lambda
\end{array}
$$

where

$$
\begin{gathered}
\tilde{A}:=\left[\begin{array}{lll}
A A^{2} & \cdots & A^{N-1}
\end{array}\right]^{T} \\
\tilde{B}:=\left[\begin{array}{cccc}
B & 0 & \cdots & 0 \\
A B & B & \ddots & \vdots \\
\vdots & \ddots & \ddots & 0 \\
A^{N-2} B & A^{N-3} B & \cdots & B
\end{array}\right] \\
\tilde{L}:=\left[\begin{array}{cccc}
L & 0 & \cdots & 0 \\
A L & L & \ddots & \vdots \\
\vdots & \ddots & \ddots & 0 \\
A^{N-2} L & A^{N-3} L & \cdots & L
\end{array}\right]
\end{gathered}
$$

Hence, we can transform the minimax problem (9) to

$$
\min _{U} \gamma
$$

subject to

$$
\begin{gathered}
\max _{W, \Lambda} \Pi \leq \gamma \\
w^{T}(k+j) P_{w} w(k+j) \leq 1 \\
\eta^{T}(k+j) P_{\eta} \eta(k+j) \leq 1 \\
u^{T}(k+j) P_{u} u(k+j) \leq 1 \\
(j=0, \cdots, N-1)
\end{gathered}
$$

where $\gamma>0$ (scalar parameter) and where;

$$
\begin{gathered}
\Pi:=\|\tilde{A} x(k)+\tilde{B} U+\tilde{L} W+\Lambda\|_{\hat{Q}}^{2}+\|U\|_{\hat{R}}^{2}, \\
\hat{Q}:=\left[\begin{array}{lll}
Q & & 0 \\
& \ddots & \\
0 & & Q
\end{array}\right], \hat{R}:=\left[\begin{array}{lll}
R & & 0 \\
& \ddots & \\
0 & & R
\end{array}\right]
\end{gathered}
$$

To eliminate the maximaization procedure, we have to remove $W$ and $\Lambda$ terms in the first constraint. For this, in the first place, following basis for all variables and transformation matrices are defined.

$$
\begin{gathered}
\zeta=\left[x(k) W^{T} \Lambda^{T} 1\right]^{T} \\
U=H_{u} \zeta\left(H_{u}:=[F \tilde{A} F \tilde{L} F 0]\right) \\
Y=H_{y} \zeta\left(H_{y}:=[C \tilde{A} C \tilde{L} I 0]\right) \\
\Lambda=H_{\eta} \zeta\left(H_{\eta}:=[00 I 0]\right) \\
1=\left(H_{1} \zeta\right)^{T}\left(H_{1} \zeta\right)\left(H_{1}:=[0 \cdots 01]\right)
\end{gathered}
$$

where

$$
F:=\left[\begin{array}{ccccc}
-F_{k} & 0 & & \cdots & 0 \\
0 & -F_{k+1} & & \ddots & \vdots \\
\vdots & \ddots & \ddots & & 0 \\
0 & \cdots & 0 & -F_{k+N-1}
\end{array}\right]
$$

By using these, we can express the first constraint condition of problem (13);

$$
\max _{W, \Lambda}\left\{\left\|H_{y} \zeta\right\|_{\hat{Q}}^{2}+\left\|H_{u} \zeta\right\|_{\hat{R}}^{2}\right\} \leq\left(H_{1} \zeta\right)^{T} \gamma\left(H_{1} \zeta\right)
$$

Please take notice that both the left side and the right side of this inequality are expressed by the quadratic forms and they have positive scalar values. Hence, if the inequality is hold by maximum values of $W$ and $\Lambda$ in left side, this inequality must be hold by any other values of them. This fact means that we can eliminate the maximization procedure in the first constraint. We can only check the following condition instead of the first constraint of problem (13).

$$
\left\|H_{y} \zeta\right\|_{\hat{Q}}^{2}+\left\|H_{u} \zeta\right\|_{\hat{R}}^{2} \leq\left(H_{1} \zeta\right)^{T} \gamma\left(H_{1} \zeta\right)
$$

In the second place, $\$ H_{-}\{w\}(j) \$$ is defined. This matrix pick out the suitable block from $W$ and satisfy the relation of $w(k+j)=H_{w}^{(j)} \zeta(j=0, \cdots, N-1)$. Then, we can derive

$$
\left(H_{w}^{(j)} \zeta\right)^{T} P_{w}\left(H_{w}^{(j)} \zeta\right) \leq\left(H_{1} \zeta\right)^{T}\left(H_{1} \zeta\right)
$$

For the constraints of $\eta$ and $u$, we can derive the following relations in the same way.

$$
\begin{aligned}
& \left(H_{\eta}^{(j)} \zeta\right)^{T} P_{\eta}\left(H_{\eta}^{(j)} \zeta\right) \leq\left(H_{1} \zeta\right)^{T}\left(H_{1} \zeta\right) \\
& \left(H_{u}^{(j)} \zeta\right)^{T} P_{u}\left(H_{u}^{(j)} \zeta\right) \leq\left(H_{1} \zeta\right)^{T}\left(H_{1} \zeta\right)
\end{aligned}
$$

Then, by using (14)-(19), all constraints in minimax problem (13) can be transformed into:

$$
\begin{gathered}
\zeta^{T}\left(H_{1}^{T} \gamma H_{1}-H_{y}^{T} \hat{Q} H_{y}-H_{u}^{T} \hat{R} H_{u}\right) \zeta \geq 0 \\
\zeta^{T}\left(H_{1}^{T} H_{1}-\left(H_{w}^{(j)}\right)^{T} P_{w} H_{w}^{(j)}\right) \zeta \geq 0 \\
\zeta^{T}\left(H_{1}^{T} H_{1}-\left(H_{\eta}^{(j)}\right)^{T} P_{\eta} H_{\eta}^{(j)}\right) \zeta \geq 0 \\
\zeta^{T}\left(H_{1}^{T} H_{1}-\left(H_{u}^{(j)}\right)^{T} P_{u} H_{u}^{(j)}\right) \zeta \geq 0 \\
(\forall \zeta \neq 0)
\end{gathered}
$$

We can transform the original minimax problem (9) to the following one by using $S$-procedure [27].

$$
\min _{F} \gamma
$$


subject to

$$
\begin{aligned}
& H_{1}^{T} \gamma H_{1}-H_{y}^{T} \hat{Q} H_{y}-H_{u}^{T} \hat{R} H_{u} \\
& -\sum_{j=0}^{N-1}\left[\tau_{j}^{w} S_{j}^{w}+\tau_{j}^{\eta} S_{j}^{\eta}+\tau_{j}^{u} S_{j}^{u}\right] \leq \gamma,
\end{aligned}
$$

where

$$
\begin{aligned}
& S_{j}^{w}=\left(H_{1}^{T} H_{1}-\left(H_{w}^{(j)}\right)^{T} P_{w} H_{w}^{(j)}\right) \\
& S_{j}^{\eta}=\left(H_{1}^{T} H_{1}-\left(H_{\eta}^{(j)}\right)^{T} P_{\eta} H_{\eta}^{(j)}\right) \\
& S_{j}^{u}=\left(H_{1}^{T} H_{1}-\left(H_{u}^{(j)}\right)^{T} P_{u} H_{u}^{(j)}\right)
\end{aligned}
$$

and where $\tau_{j}^{w}, \tau_{j}^{\eta}, \tau_{j}^{u}$ are positive semi-definite scalars. It must be noted that this transformation satisfies only a sufficient condition of $S$-procedure, since $S$-procedure is not the so-called "lossless" in this case. We cannot therefore avoid that the design results are slightly conservative. Nevertheless, we can expect the reduction of conservativeness in design result by this technique in contrast with the results by preexisting methods. Because the conservativeness caused by $S$-procedure is too small to put a matter for practical purposes.

Finally, using "Schur-complement" [28], we can transformed the minimization problem (9) into the following problem which can be solved easily by using some optimization tool.

$$
\min _{F} \gamma
$$

subject to

$$
\begin{aligned}
& {\left[\begin{array}{ccc}
H_{1}^{T} \gamma H_{1}-\Sigma & H_{y}^{T} & H_{u}^{T} \\
H_{y} & \hat{Q}^{-1} & 0 \\
H_{u} & 0 & \hat{R}^{-1}
\end{array}\right] \geq 0} \\
& \tau_{j}^{w}, \tau_{j}^{\eta}, \tau_{j}^{u} \geq 0
\end{aligned}
$$

where

$$
\Sigma:=\sum_{j=0}^{N-1}\left[\tau_{j}^{w} S_{j}^{w}+\tau_{j}^{\eta} S_{j}^{\eta}+\tau_{j}^{u} S_{j}^{u}\right]
$$

\section{MPC with Adaptive DA Converter}

\subsection{Interpolation of Control Inputs}

Although the information about future sampling points is need for getting the current control input by the interpolating operations of the sampled-data control systems, it's impossible to obtain them. Therefore, we have been forced to tolerate the long time-delay during the DA conversion to wait for getting the indispensable informa- tion.

However, in the case of controlling the systems with relatively fast-moving dynamics, such as robots or vehicles, the method with long time-delay is unable to be applied. Furthermore, it takes much computation time to calculate the interpolation by using the high-order sampling functions to DA conversion in sampled-data control system.Therefore a new idea to use the predictive control inputs obtained by MPC for interpolation is proposed.

In MPC algorithm, the optimal control inputs $\{\hat{u}(k \mid k), \cdots, \hat{u}(k \mid k+N-1)\}$ are calculated in each step, and only the first control input $\hat{u}(k \mid k)$ is used as a real control input.Therefore, we consider to use the other optimal control inputs $\{\hat{u}(k \mid k+1), \cdots\}$ as virtual future sampling points.Actually, it is only necessary to use the optimal control inputs which are needed for interpolation according to the sampling function.

Figure 4 shows this way using the 2 nd order spline function for interpolations. Only $\hat{u}(k \mid k+1)$ is used as a virtual future sampling point in this case. By using the predictive control inputs for interpolation, it becomes possible to reduce the time-delay in the DA conversion, and the total time-delay to be needed is just only computation time of optimization in current step.

Of course, it needs to take account that there is a difference between virtual future sampling points and real sampling points like $\hat{u}(k+1 \mid k) \neq \hat{u}(k+1)$ in future step. However, we consider that this point is not a critical problem because the influence on interpolated waveform due to prediction error is not so big compared to the scale of prediction error. Although the differentiability of each sampling function is lost at sampling points, this also does not become a critical problem compared to the

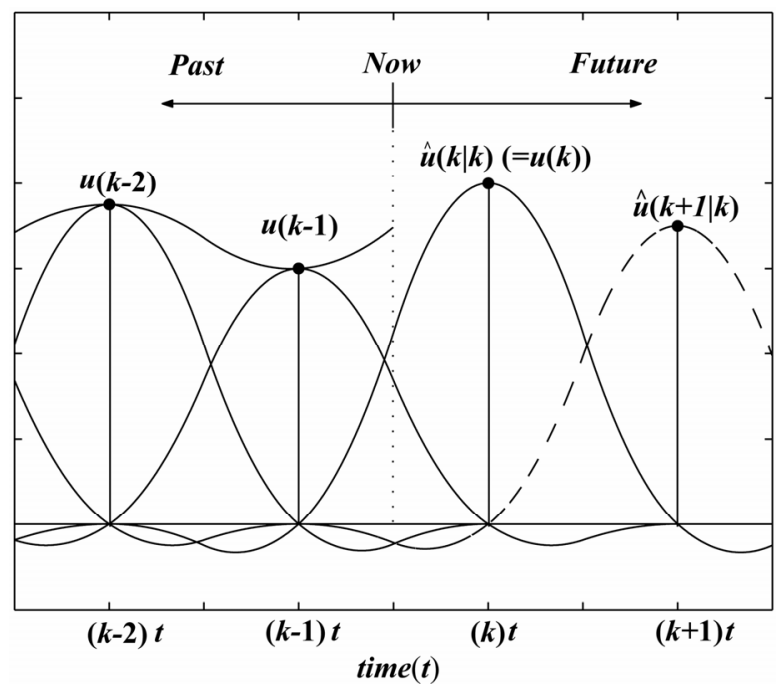

Figure 4. Interpolation based on a 2nd order spline sampling function using predictive future control inputs. 
zero-order hold, and it is possible to keep a certain level of smoothness.

\subsection{Adaptive DA Converter}

The spline functions provide various samplingfunctions with all kinds of orders. Therefore, we consider switching the spline functions optimally according to the system status in the adaptive DA converter. In this paper, we use the spline functions with the order $m=0,1,2$ as sampling functions. Namely, in the case of $m=0$, the sampling function is equivalent to the staircase function. In the case of $m=1$, it's the 1 st order piecewise polynomial function, and in $m=2$, 2nd order one as shown in Figure 5.

Appropriate selecting the values of $m$ according to the object, enables to deal with DA conversion flexibly and precisely in the interpolation operation. Although the interpolation is more precisely in the case of using the spline function with $m=3$ or more, it's difficult to apply to fast-moving dynamic systems due to the bigger amount of calculation. Therefore we use only the spline functions with the order $m=0,1,2$.

The interpolated signals in the closed-open interval $[k \tau$, $(k+1) \tau)$, using these sampling functions are obtained as follows,

$$
u(t)=\sum_{l=k}^{k+1}\left\{u(l) \cdot{ }^{1,2} \psi(t-l \tau)\right\} \quad(m=0,1)
$$

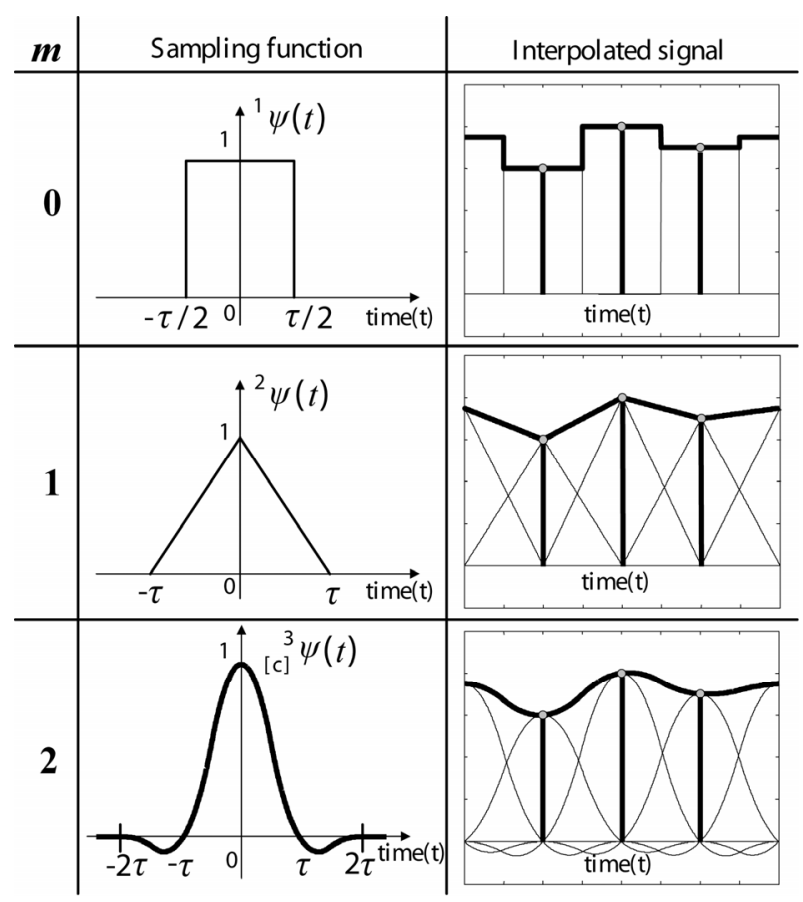

Figure 5. Sampling functions and their interpolations with $m=0,1,2$ ( $\tau$ is sampling interval).

$$
u(t)=\sum_{l=k}^{k+2}\left\{u(l) \cdot{ }^{3} \psi(t-l \tau)\right\}(m=2)
$$

where $u(t)$ and $u(l)$ are analog signal and digital signal respectively, and $\tau$ is sampling interval.

The interval to be interpolated is also divided to $d$ sections, and the dividing points $u_{m}(j ; k),(j=1,2, \cdots, d-1)$ on interpolated waveforms are used for the selection of parameter $m$, that indicates the degree of spline sampling functions. Figure 6 shows the difference of the interpolation and dividing points according to the sampling function with $m=0,1,2$ and $d=5$.

The calculation of the dividing points $u_{m}(j ; k)$ as follows,

$$
\begin{array}{r}
u_{m}(j ; k)=\sum_{l=k-\alpha}^{k+\alpha-1}\left\{u(l) \cdot{ }^{m} \psi\left((k-1) \tau+\frac{\tau}{d} \cdot j-l \tau\right)\right\} \\
(j=1,2, \cdots ; d=1)
\end{array}
$$

where $\alpha$ is the number of samples which the sampling function needs for interpolation, and it is adjusted according to the sampling function.

From several test simulation results, we have obtained that it most appropriate to set the divided number of interval, $d=5$ due to the trade-off of computation time and precision. If $d=5$, the calculation amount in the adaptive DA converter is also vanishingly small compared to the calculation in MPC controller keeping a certain level of accuracy. Then, we summarize the algorithm to switch the spline sampling functions for the adaptive DA converter as follows,

(step 1) Set step $k=0$.

(step 2) The dividing points $u_{m}(j ; k)$ are calculated.

(step 3) The predicted state values $x_{m}(j+1 ; k)$ in this interval are calculated using internal model of DA converter and the dividing points $u_{m}(j ; k)$.

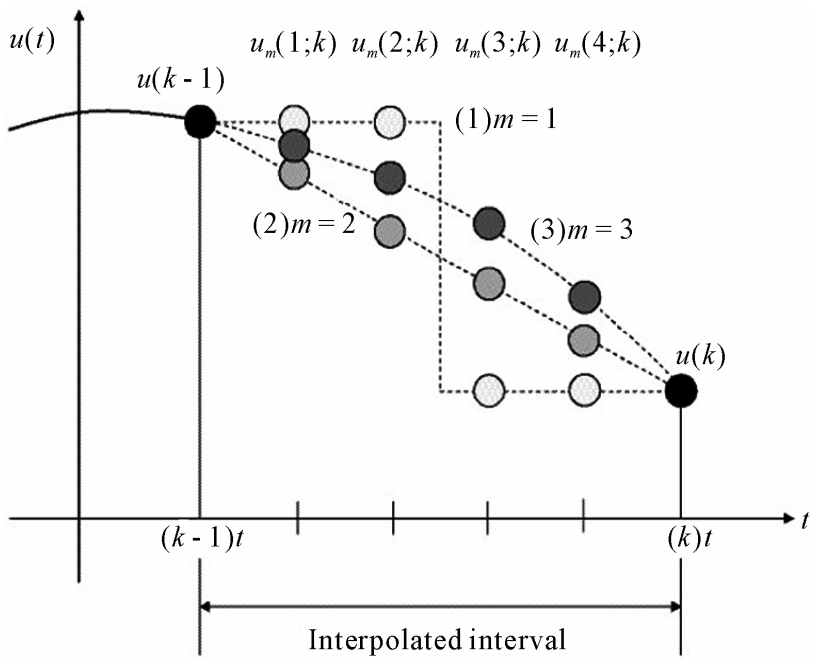

Figure 6. Interpolation ways $(d=5)$. 
(step 4) If the interpolation waves exceeds the constrained conditions of control input due to the overshoot or undershoot, this $m$ is excluded.

(step 5) The evaluation values of evaluation function $J(k)$ od Equation (8) are calculated in each $m$.

(step 6) The parameter $m$ whose evaluation value is the smallest is selected as an interpolation way in this interval, and then $k=k+1$ and go back to (step1).

\section{Numerical Experiments}

The experimental conditions are summarized as follows.

- The robotic wheelchair goes straight according to the reference path usually.

- The signal from the BMI was read at constant intervals (100 ms).

- The signal classified the three types: 0 (the signal none), 1 or -1 (left or right evasion). 1 and -1 are the emergent evasion signals against the obstacle appeared suddenly. Each value of signals 1 and -1 has $0-1$.
- If the signal 1 or -1 is detected, the wheelchair does the evasion run in a specified direction according to half oval orbit. The radius of half oval changes according to value of the signal.

Now we assume the following perturbations of $l$ and $c$ in the wheelchair model.

$$
\begin{aligned}
& l \in\{l \mid 0.08 \leq l \leq 0.12\}, \\
& c \in\{c \mid 0.03 \leq c \leq 0.07\}
\end{aligned}
$$

The weights of the cost function in Equation (8) are

$$
Q=\left[\begin{array}{cc}
125 & 0 \\
0 & 15
\end{array}\right], R=\left[\begin{array}{cc}
0.55 & 0 \\
0 & 0.15
\end{array}\right]
$$

Figures 7-10 show simulation results with various conditions. The parameter $r=0.2$ [m]. The size of obstacle is different between Figures $\mathbf{7}$ and $\mathbf{8}$, but initial position of obstacle is same. Figs. 9and 10 show the two obstacle case. In Figures 7-9, red line indicates the nearest trajectory of the center of gravity (C.G.) of robotic wheel

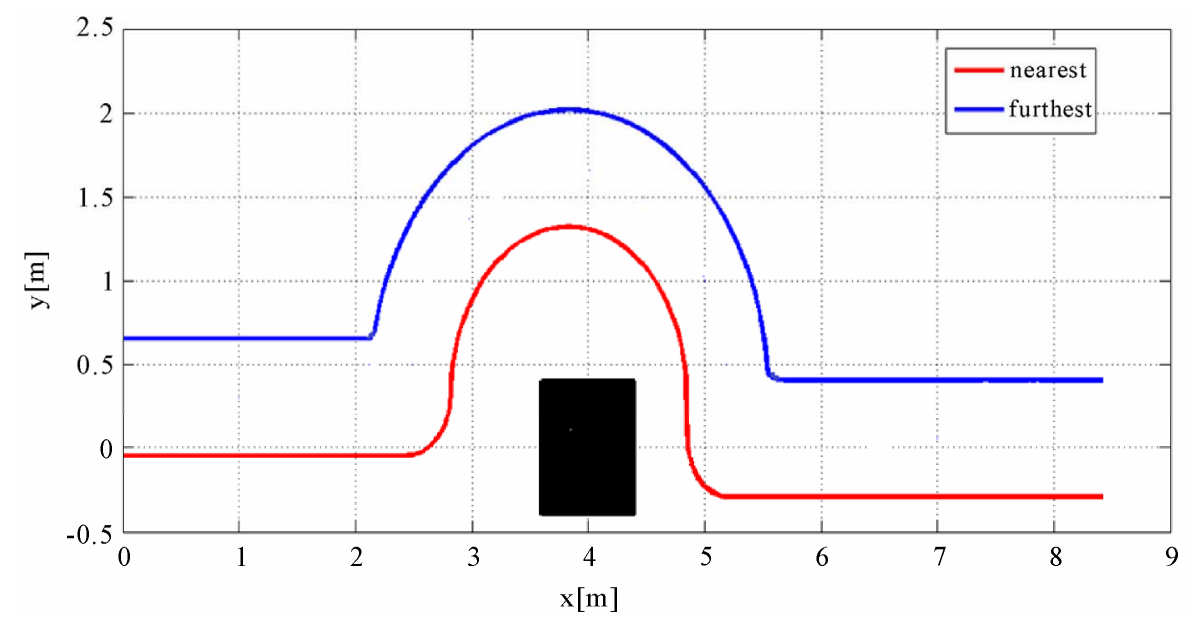

Figure 7. The C.G. trajectories (a).

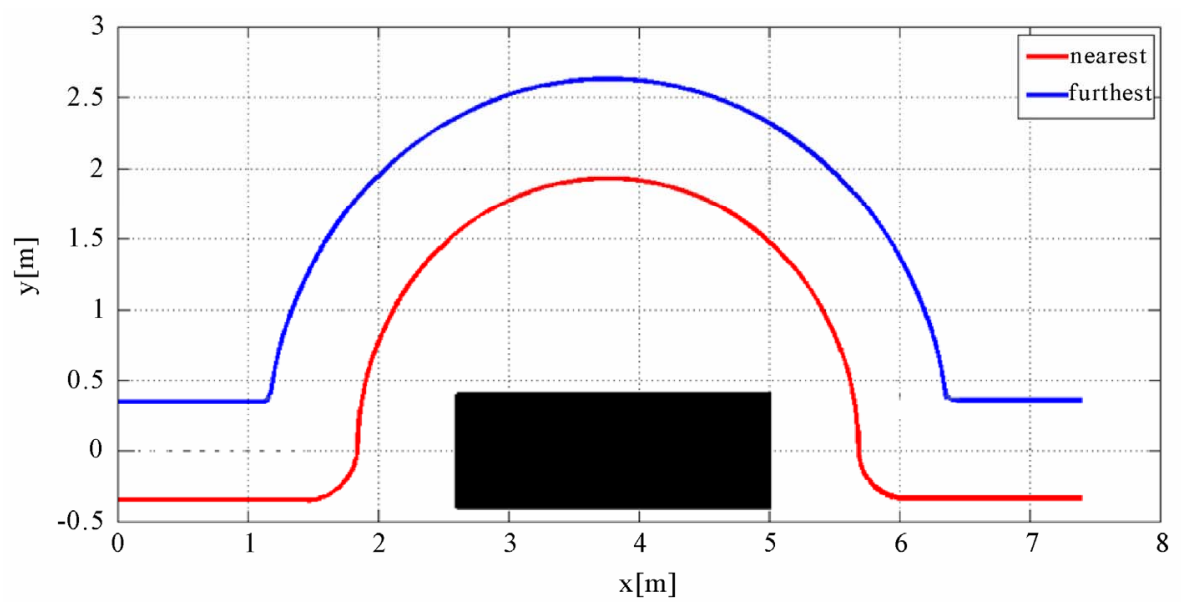

Figure 8. The C.G. trajectories (b). 


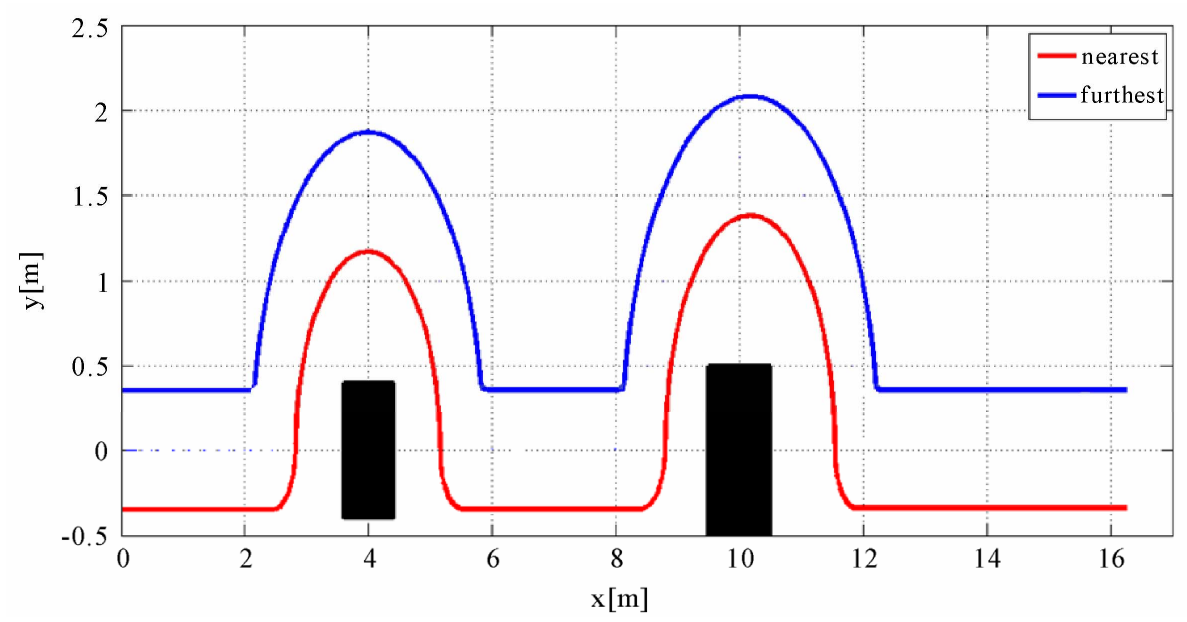

Figure 9. The C.G. trajectories (c).

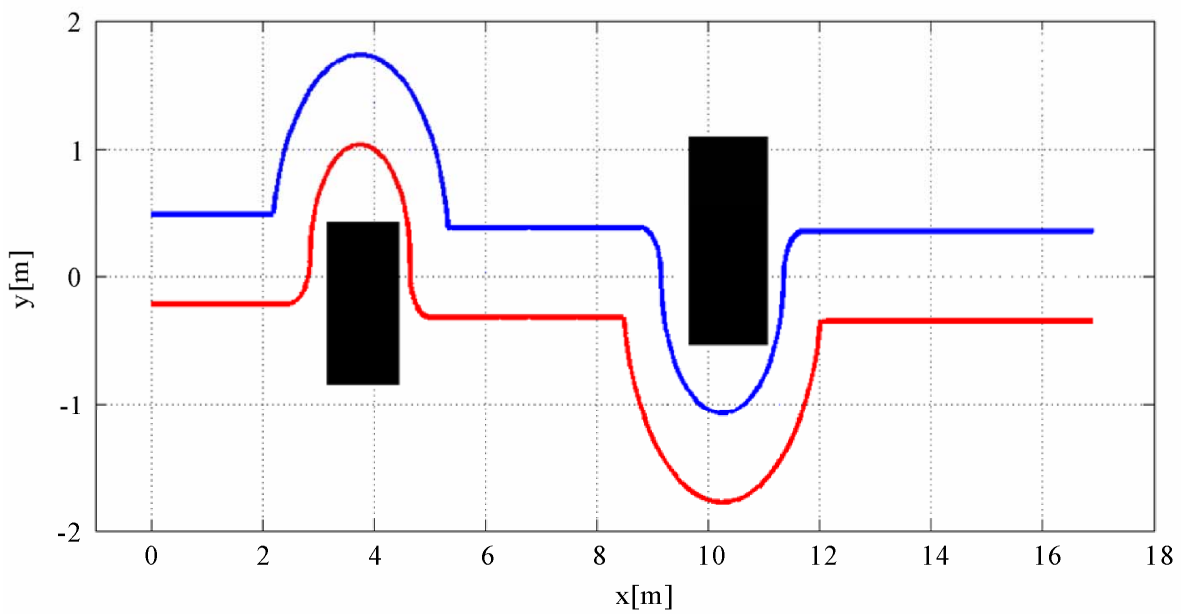

Figure 10. The C.G. trajectories (d).

chair to the obstacle against the perturbation of model uncertainties. Blue line indicates the furthest one. All results show that thewheelchair can avoid the obstacle safely even if parameter uncertainties are existing. For the comparison, in the case of using the standard MPC instead of the proposed minimax MPC, the wheelchair had collided with the obstacle whenever the parameters $(l$ and $c$ ) were perturbed even if no collision occurred with the nominal values of $l$ and $c$. Since the standard MPC is a nominal control method and it cannot guarantee the robustness property against the parameter perturbations.Hence, we can easily see that the proposed method have good robust performance against the model uncertainties and we can recognize the effectiveness of the proposed method.

\section{Conclusions}

As the first stage of the development of the BMI based wheelchair system, new minimax robust MPC method with the adaptive DA converter applied to the obstacle avoidance system in the BMI based wheelchair has been proposed. Simulation results have been illustrated to indicate the good robust performance as the development of the former stage of real experimental system. From these results, the proving test by the real experiments of the BMI based wheelchair by the real experiments will be done in next stage.

In addition to, the proposed minimax MPC method is easily extended the systems with other constraints which are specified by ellipsoidal bounds, for example, state estimation errors and so on as follows. In the case that $x(k)$ is not full measured and we need to estimate $x(k)$, where the bound of estimation error $\mathrm{e}(k)=x(k)-\hat{x}(k)$ is guaranteed an ellipsoidal set as:

$$
\mathrm{e}^{T}(k) P_{e} \mathrm{e}(k) \leq 1
$$

where, $P_{e}$ is a positive symmetric matrix for weight. This 
specification of estimation error is standard one. Now we introduce $H_{e}$ as:

$$
H_{e}:=[10 \cdots 0-\hat{x}(k)]
$$

then the relation of $e(k)=H_{e} \zeta$ is hold. And the condition below is also hold.

$$
\zeta^{T}\left(H_{1}^{T} H_{1}-\left(H_{e}^{(j)}\right)^{T} P_{e} H_{e}^{(j)}\right) \zeta \geq 0
$$

Since this condition has same form as other constraints in Equation (24), we can include this condition into the condition of problem (25) by using a new variable $\tau_{j}^{e}$.

\section{References}

[1] C. E. Garcia, D. M. Prett and M. Morari, "Model Predictive Control: Theory and Practice-A Survey," Automatica, Vol. 25, No. 3, 1989, pp. 335-348. doi:10.1016/0005-1098(89)90002-2

[2] J. Ackermann, "Sampled-Data Control Systems: Analysis and Synthesis, Robust System Design," Springer-Verlag, Berlin, 1985.

[3] P. T. Kabamba, "Control of Linear Systems Using Generalized Sampled-Data Hold Functions," IEEE Transactions on Automatic Control, Vol. 32, No. 9, 1987, pp. 772-783. doi:10.1109/TAC.1987.1104711

[4] B. D. O. Anderson, "Controller Design: Moving from Theory to Practice," IEEE Control Systems Magazine, Vol. 13, No. 4, 1983, pp. 16-25. doi:10.1109/37.229554

[5] Y. Yamamoto, "A Function Space Approach to SampledData Control Systems and Tracking Problems," IEEE Transactions on Automatic Control, Vol. 39, No. 4, 1994, pp. 703-713. doi:10.1109/9.286247

[6] D. R. Ramirez and E. F. Camacho, "Piecewise Affinity of Min-Max MPC with Bounded Additiveuncertainties and a Quadratic Criterion," Automatica, Vol. 42, No. 2, 2006, pp. 295-302. doi:10.1016/j.automatica.2005.09.009

[7] A. Grancharova and T. A. Johansen, "Computation, Approximation and Stability of Explicit Feedback Min-MaxNonlinear Model Predictive Control," Automatica, Vol. 45, No. 5, 2009, pp. 1134-1143. doi:10.1016/j.automatica.2008.12.023

[8] P. J. Campo and M. Morari, "Robust Model Predictive Control," Proceedings of 1987 American Control Conference, Minneapolis, 10-12 June 1987, pp. 1021-1026.

[9] Z. Q. Zheng and M. Morari, "Robust Stability of Constrained Model Predictive Control," Proceedings of 1987 American Control Conference, San Francisco, 2-4 June 1993, pp. 379-383.

[10] M. V. Kothare, V. Balakrishnan and M. Morari, "Robust Constrained Model Predictive Control using Linear MatrixInequalities," Automatica, Vol. 32, No. 10, 1996, pp. 1361-1379. doi:10.1016/0005-1098(96)00063-5

[11] F. A. Cuzzola, J. C. Geromel and M. Morari, "An Improved Discrete-Time Robust Approach for Constrained Model Predictive Control," Proceedings of 2001 Euro- pean Control Conference, Porto, 4-7 September 2001, pp. 2759-2764.

[12] J. C. Allwright and G. C. Papavasiliou, "On Linear Programming and Robust Model-Predictive Control Using Impulse-Responses," System \& Control Letters, Vol. 18, No. 2, 1992, pp. 159-164.

doi:10.1016/0167-6911(92)90020-S

[13] J. H. Lee and Z. Yu, "Worst-Case Formulation of Model Predictive Control for Systems with Bounded Parameters," Automatica, Vol. 33, No. 5, 1997, pp. 768-781. doi:10.1016/S0005-1098(96)00255-5

[14] A. Bemporad and M. Morari, "Robust Model Predictive Control: A Survey," In: A. Garulli, A. Tesi and A. Vicino, Eds., Robustness in Identification and Control, Lecture Notes in Control and Information Sciences, Vol. 245, Springer-Verlag, 1999, pp. 207-226.

[15] A. Bemporad and A. Garulli, "Output-Feedback Predictive Control of Constrained Linear Systems via Set-Membership State Estimation," International Journal of Control, Vol. 73, No. 8, 2000, pp. 655-665.

doi: $10.1080 / 002071700403420$

[16] P. O. M. Scokaert and D. Q. Mayne, "Min-Max Feedback Model Predictive Control for Constrained Linear Systems," IEEE Transactions on Automatic Control, Vol. 43, No. 8, 1998, pp. 1136-1142. doi:10.1109/9.704989

[17] D. Q. Mayne, J. B. Rawlings, C. V. Rao and M. Scokaert, "Constrained Model Predictive Control: Stability and Optimality," Automatica, Vol. 36, No. 6, 2000, pp. 789-814. doi:10.1016/S0005-1098(99)00214-9

[18] S. Waldert, T. Pistohl, C. Braun, T. Ball, A. Aertsen and C. Mehring, "A Review on Directional Information in Neural Signals for Brain-Machine Interfaces," Journal of Physiology Paris, Vol. 103, No. 3-5, 2009, pp. 244-254. doi:10.1016/j.jphysparis.2009.08.007

[19] R. Heliot and J. M. Carmena, "Brain-Machine Interfaces," Encyclopedia of Behavioral Neuroscience, 2010, pp. 221-225.

http://www.sciencedirect.com/science/article/pii/B97800 80453965002098

[20] T. N. Lal, M. Schroder, T. Hinterberger, J. Weston and M. Bogdan, "Support Vector Channel Selection in BCI," IEEE Transactions on Biomedical Engineering, Vol. 51, No. 6, 2004, pp. 1003-1010. doi:10.1109/TBME.2004.827827

[21] J. del R. Millan, M. Franze, J. Mourino, F. Cincotti and F. Babiloni, "Relevant EEG Features for the Classification of Spontaneous Motor-Related Tasks," Biological Cybernetics, Vol. 86, No. 2, 2002, pp. 89-95. doi:10.1007/s004220100282

[22] V. N. Vapnik, "Statistical Learning Theory," John Wiley \& Sons, New York, 1998.

[23] C. T. Suand and C. H. Yang, "Feature Selection for the SVM: An Application to Hypertension Diagnosis," Expert Systems with Applications, Vol. 34, No. 1, 2008, pp. 754-763. doi:10.1016/j.eswa.2006.10.010

[24] M. Sampei and M. Ishikawa, "State Estimation and Output Feedback Control of Nonholonomic Mobile Systems 
using Time-Scale Transformation," Transactions of the Institute of Systems, Control and Information Engineers, Vol. 13, No. 3, 2000, pp. 124-133.

[25] R. Fierro and F. L. Lewis, "Control of a Nonholonomic Mobile Robot: Backstepping Kinematics into Dynamics," Journal of Robotic Systems, Vol. 14, No. 3, 1997, pp. 149-163. doi:10.1002/(SICI)1097-4563(199703)14:3<149::AID-R OB1>3.0.CO;2-R

[26] J. Tang, K. Watanabe, M. Nakamura and N. Koga, "Fuz- zy Gaussian Neural Network Controller and Its Application to the Control of a Mobile Robot," Transactions of Japan Society of Mechanical Engineers, Series C, Vol. 59, No. 564, 1993, pp. 2290-2297.

[27] S. Boyd and C. H. Barratt, "Linear Controller Design: Limits of Performance," Prentice-Hall, Upper Saddle River, 1991.

[28] K. Zhou, J. C. Doyle and K. Glover, "Robust and Optimal Control," Prentice-Hall, Upper Saddle River, 1996. 